\section{Urologia \\ Internationalis}

\section{Priyadarshi Kumar \\ Vivek Mehta \\ Vinod H. Nargund}

Department of Urology, Homerton

University and St Bartholomew's

Hospitals, London, UK
Urol Int 2010;84:125-131

DOI: $\underline{10.1159 / 000277587}$

\title{
Clinical Management of Chronic Testicular Pain
}

Key Words

Orchialgia - Testicular pain, chronic • Genitourinary surgery

\section{Abstract}

Aim: To review the causes and principles and recent concepts in the management of testicular pain. Introduction: Chronic testicular pain is a common presenting symptom in genitourinary surgery. Due to increased awareness of testicular cancer and in men's health more cases are likely to be referred. Material and Methods: A literature search was made for abstracts, original papers and review articles in the Cochrane Database, Medline and medical textbooks using the words 'testicular pain' and orchialgia to find the causes and mechanisms of testicular pain. The management and algorithm have been structured on evidence-based management strategies. Results: The management of chronic testicular pain remains essentially based on clinical assessment. In recent years there have been advances in the nonsurgical management of testicular pain mainly because of the emergence of pain relief as a specialty. However, in some cases pain control is a problem and may ultimately conclude with orchiectomy. Conclusions: The management of chronic testicular pain includes a careful assessment of testicular and extratesticular causes. Relief of symptoms is not always possible and gaining an insight into the patient's concerns and empathizing with their condition is paramount in help- ing them cope with their symptoms. Surgery should not be undertaken lightly for there is no guarantee that there will always be resolution of symptoms and the patient should be counseled accordingly.

Copyright $\odot 2010$ S. Karger AG, Basel

\section{Introduction}

Chronic testicular pain is a common presenting problem in urology and general practitioner's clinics. There seems to be an increase in such referrals due to public awareness of testicular cancer. With an increase in easily available information by the media the last decade has seen an explosion in magazines focusing on men's health and 'men's problems'. Similarly the internet abounds with easily accessible websites devoted to health concerns giving voluminous information to consult. A major side effect of this however is anxiety about one's own problems, encouraging the individual to seek further medical advice and reassurance.

Chronic orchialgia is defined as an intermittent or constant testicular pain, unilateral or bilateral, lasting for over 3 months that interferes significantly with the patient's daily activities [1]. Significant morbidity results due to interference with work and social life with psychological and financial implications as underlying diagno-

\section{KARGER}

Fax +41 613061234 E-Mail karger@karger.ch www.karger.com

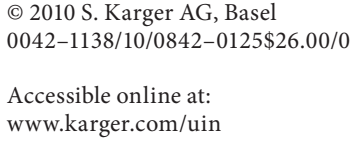

Mr. Vinod H. Nargund

St Bartholomew's Hospital

West Smithfield

London EC1A 7BE (UK)

Tel. +44 207601 8391, Fax +44 207601 7844, E-Mail vinodnargund@yahoo.co.uk 
sis is not always possible. Chronic orchialgia is not the preserve of the urologist alone and input of other disciplines like psychiatric and pain teams may be beneficial in selected cases. In this review we will look at the causes, investigations and management of chronic orchialgia. A search was performed of the Cochrane Database, Medline, PubMed and medical textbooks in the preparation of this review.

\section{Etiology}

Pain experienced in scrotal and testicular areas has varied origins including abdominal visceral causes due to its development in the abdominal cavity. During its descent in intrauterine life, the testis carries autonomic nerve supply and vasculature with it. The nerves travel along the testicular vessels to the aortic and renal plexuses. Both testis (T10-T12) and epididymis (T12-L1) are innervated from the presacral ganglia. The genital branch of genitofemoral and ilioinguinal nerve provide sensory innervation for the anterior scrotal wall. The posterior surface of the scrotum is innervated by scrotal branches of the superficial perineal nerve, via the perineal branch of the pudendal nerve (S1-S3).

In mammalian experiments, Rauchenwald et al. [2] have demonstrated additional afferent and efferent fibers to the pelvic plexus along the vas deferens with a smaller testicular contribution coming from the sacral parasympathetic nerves. There is also bilateral criss-cross innervation of the testes [3]. Testis innervation is exemplified by colicky pain in the ureter which may relate to the inner surface of the scrotum (genital branch of genitofemoral nerve L1). Similarly back pain may radiate to the testicle due to sensory nerve root irritation (T10-L1). Inguinal hernias may stretch the genitofemoral and ilioinguinal nerves causing discomfort in the scrotum and testes. Testicular pain secondary to compression or injury of the ilioinguinal, genitofemoral and iliohypogastric nerves may be caused by surgery for hernia [4].

Similarly referred pain has also been described in entrapment neuropathies of the pudendal nerve [5], tendonitis of the pubic attachment of the inguinal ligament, gluteal fibrositis, pelviureteric junction obstruction, iliac and aortic aneurysms [6]. The pudendal nerve is predisposed to entrapment causing scrotal and perineal pain. The pudendal nerve entrapment may occur at two points - firstly, at the level of ischial spine, and secondly, as it runs under the levator ani through the pudendal canal [7].
Table 1. Etiology of chronic orchialgia

\begin{tabular}{ll}
\hline Testis & Trauma \\
& Tumor \\
& Orchitis/epididymo-orchitis \\
& Torsion \\
& Atrophy \\
& Scarring \\
\hline Epididymis & Epididymitis \\
& Cysts \\
& Sperm granuloma \\
& Spermatocele \\
\hline Cord & Vasectomy \\
& Varicocele \\
& Hernia \\
& Nerve pain \\
& Iatrogenic \\
& Arteriovenous fistula \\
\hline Scrotal wall & Hydrocele \\
\hline Urethral & Benign prostatic hyperplasia \\
& Prostatitis (acute and chronic) \\
\hline Urinary tract & Urethral stricture \\
\hline Exychological & Stone \\
& Infection \\
\hline & Radiculitis \\
\hline Irritable bowel syndrome \\
\hline
\end{tabular}

Other causes of testicular pain could be classified according anatomical origin: epididymal, testicular, cordrelated, and scrotal. The causes could be inflammatory, infective, neoplastic, traumatic, and iatrogenic. All these origins of pain are summarized in table 1.

\section{Clinical Assessment}

The key to successful assessment remains the history and establishing a good rapport with the patient. The evaluation will diagnose underlying causes and assess the effect of the condition on the patient's daily life and mental state. Pain is assessed with regard to site, laterality, intensity, duration, type, radiation, aggravating and relieving factors. The character of the pain may vary from an acute intermittent sharp pain to a dull constant ache. Aggravating and relieving factors need assessment, for example activities such as touching (hyperesthesia), sitting, lifting heavy materials and intercourse. Wearing 
tight underwear may aggravate or relieve the pain. Relief of pain may be related to analgesia and posture.

It is important to enquire and look for any associated lumps. Contrary to popular belief a fair proportion of testicular tumors do present with pain. If any abnormal swelling is noted, scrotal ultrasound is necessary to exclude neoplasm. Trauma-induced pain may alert the patient more to the testicular symptoms in the presence of a neoplasm due to hemorrhage into the tumor.

Chronic orchitis or epididymitis is associated with pain and swelling of the testis may be due to accompanying lower urinary tract symptoms. There may be a preceding history of sexually transmitted diseases. It is also important to know whether testicular pain is part of chronic prostatitis/chronic pelvic pain syndrome. In these patients there may be a history of associated perineal, suprapubic, penile or ejaculatory pain. In orchitis, the testis itself is globally tender and may feel quite hard like a neoplasm, and again an urgent scrotal ultrasound is recommended. Epididymitis will reveal a tender and enlarged epididymis. Recurrent epididymo-orchitis may lead to low-grade pain due to scarring. In chronic epididymo-orchitis, particularly due to tuberculosis, pain may not be the main feature. Intermittent torsion of the testicle may also present as chronic orchialgia. The history will elicit recurrent episodes of acute scrotal pain and testes may have a horizontal lie. This is principally a clinical diagnosis and exploration and fixation is advisable to prevent further torsion, subsequent pain and gangrene of the testis.

A hydrocele or epididymal cyst would be described by the patient as a swelling of the testicle associated with or without a dull ache. Impalpable testis due to tense hydrocele can be visualized with ultrasound. Large epididymal cysts may be confused with a hydrocele and again sonography will differentiate the two. The other differentials of an extratesticular lesion include sperm granuloma and spermatocele.

It is useful to examine the patient in the standing and lying down positions as entities like hernia and varicocele are better visualized in the standing position. The hernial orifices are examined for occult hernia. Getting above the swelling is not possible in inguinoscrotal hernias due to the increased thickness of the cord. If the patient is experiencing intermittent obstruction in the hernia, there may be radiation of pain felt in periumbilical region. Testicular pain can result from nerve entrapment or cord fibrosis due to previous groin surgery.

Pain following vasectomy is due to epididymal engorgement and is present in $33 \%$ of patients 4 years after vasectomy with only $15 \%$ finding the pain troublesome [8]. Pain may be a continuous or intermittent dull ache and even periodic in nature with tenderness and distension of epididymis.

Varicoceles may cause dragging pain on standing and relief is obtained by lying down. They also feel like a 'bag of worms' and are seen better on standing. Occasionally arteriovenous fistula in the cord may cause testicular pain [9].

As already mentioned, lower urinary tract symptoms due to bladder outflow obstruction, urethral stricture and prostatitis may cause testicular pain. A history of sexually transmitted diseases may be significant in diagnosing prostatitis and urethral stricture. Digital rectal examination helps to evaluate prostatic hypertrophy, and the presence of tenderness or edema indicates prostatitis.

A history of abdominal and vague musculoskeletal symptoms may suggest irritable bowel syndrome or aneurysms. Radiculitis-induced scrotal pain may be associated with positive neurological examination.

\section{Investigations}

\section{Urine Analysis}

All patients should have urine tested at least by a dipstick or by microscopy followed by urine culture where indicated. A fair proportion of patients with orchitis, epididymitis, and prostatitis have negative cultures. In suspected cases of prostatitis, lower urinary tract localization studies are of limited value as only $10 \%$ of chronic prostatitis patients have bacterial infection [10]. However, a definitive diagnosis is obtained with the Meares-Stamey four-glass test, or the two-glass test (pre- and postmassage screen), helps to differentiate between chronic bacterial prostatitis and chronic pelvic pain syndrome.

\section{Imaging}

Ultrasound of Testes. The most reliable imaging modality in the management of chronic testicular pain is ultrasonography of the testis and inguinal region. This is a particularly useful tool in diagnosing intrascrotal lesions that are not impalpable. Lau et al. [11] observed that in patients with testicular pain of $>14$ days duration sonography detected lesions in $38 \%$ of patients with no clinical findings with a sensitivity of $71.4 \%$ and specificity of $90.9 \%$.

Abdominal Ultrasound. Patients with symptoms of voiding dysfunction need bladder ultrasonography and 
uroflowmetry to assess bladder emptying. In these patients voiding charts and cystometric studies are also useful adjuncts.

Transrectal Ultrasonography. Transrectal ultrasound plays a limited role in prostatitis, although it may help to rule out underlying prostatic calculi, congenital cysts or collections.

Computed Tomography. Computed tomography with or without urography will help to detect urolithiasis and other abdominal conditions.

Magnetic Resonance Imaging. MRI plays a role in the diagnosis of radiculitis. It has also been applied in recent years to imaging of the scrotum $[12,13]$ and sonographically indeterminate testicular lesions [14]. MRI also enhances the certainty of the diagnosis of malignancy of nonpalpable testicular lesions, particularly in conditions that generally give equivocal diagnosis with ultrasonography. MRI allows characterization of scrotal masses as intratesticular or extratesticular. It also demonstrates various types of lesions and tissue, including cysts or fluid, solid masses, fat, and fibrosis.

\section{Management}

From the outset it is important to be honest with the patient and make him understand that there is no 'magic cure' thereby gaining the patient's trust. $\mathrm{He}$ is also made aware that further consultations, investigations and interventions may be necessary. The financial implications of chronic orchialgia are significant in terms of work time lost, and therefore careful and meticulous note taking is necessary as these records are likely to be scrutinized if the patient is seeking financial compensation. One of the utmost priorities is the exclusion of a testicular neoplasm and ultrasound is therefore advisable in all patients.

The management depends on the cause or presumed etiology of testicular pain. It is often the case, however, that no particular cause is found. The management of idiopathic pain will be discussed later. If there is clinical, microbiological or radiological evidence of epididymitis, orchitis or prostatitis, then a combination therapy of doxycycline with a fluoroquinolone will cover most organisms. Empirical treatment with broad-spectrum antimicrobials is useful if the diagnosis of prostatitis is clinically suspected. The fluoroquinolones are the first choice of therapy for chronic bacterial prostatitis, in particular levofloxacin which is as effective as ciprofloxacin but shows a better prostatic penetration and is given once daily [10].
$\alpha$-Adrenoceptor-blocking agents like tamsulosin, doxazosin or alfuzosin may be used in the presence of bladder outflow obstruction and prostatitis. If an intrascrotal lesion, such as hydrocele, spermatocele or varicocele, is found, then surgical treatment may be offered with a good chance of success [15]. It is imperative to stress that surgery does not necessarily improve orchialgia.

Management of inguinal hernia depends on the severity of symptoms and type of hernia. Post-hernia groin and testicular pain may be managed by injection therapy, removal of the mesh and ilioinguinal nerve transaction by laparoscopic or by open approach [16]. The success of surgical treatment of post-vasectomy pain is dependent on careful selection of patients and operative intervention. Nearly a third of patients develop post-vasectomy orchialgia, but $<50 \%$ of these patients require intervention [8]. Alleviation of pain is possible with excision of vasectomy nodules. Similarly, epididymectomy resolves pain in $95 \%$ of cases with painful epididymides [17]. The probable mechanism of this relief is due to excision of obstructed, granulomatous and fibrotic epididymis [18]. Intermittent torsion of the testicle is a clinical diagnosis and is managed by fixation of both testes.

If the pain is extratesticular in origin, then a referral to the appropriate specialist is made for further management, e.g. vascular surgeons for aneurysms, gastroenterologists for irritable bowel, or orthopedic surgeons for vertebral disease.

\section{Idiopathic Testicular Pain}

If no cause is found after full and thorough assessment, a referral to 'pain clinic' may be imperative. Pain clinics offer the expertise in nonsurgical management including interventional therapy, pharmacotherapy, and psychotherapy centered around pain management programs.

\section{Pharmacological Treatment}

Appropriate selection and titration of analgesic drugs is necessitated by: (1) the different etiologies and mechanisms of pain, (2) wide variation in reported symptoms (symptom heterogeneity) arising from similar degrees of pathology, and (3) very large inter-patient variability in response to analgesic drugs.

The established principle of a step-wise increase in analgesic strength (from simple analgesics to opioids) in the World Health Organization's three-step analgesic ladder was first published in 1986 and subsequently revised in 1996 [19] (fig. 1). The guidelines also emphasize the ben- 
efits of multimodal therapy by recommending the concomitant use rather than substitution of simple analgesics and adjuvant medications at each step.

Paracetamol and Non-Steroidal Anti-Inflammatory Analgesic Drugs. Paracetamol, within its dose recommendations, is a very safe and effective analgesic; paracetamol and non-steroidal anti-inflammatory drugs (NSAIDs) being devoid of central depressant or moodaltering effects are ideal either alone or in combination and provide multimodal analgesia. As these agents have a ceiling effect, they require careful titration with respect to their renal, hepatic and gastrointestinal side effect profile. NSAIDs in particular are useful as the main analgesic agents for moderate pain, or for adjuvant therapy with opioids being used for severe pain. However, their longterm usage is fraught with problems associated with nonspecific cyclooxygenase (COX-1) inhibition which could lead to loss of gastric cytoprotection, renal homeostasis, and platelet dysfunction. It is therefore advisable to use them sparingly and on a short-term basis.

Adjuvant Drugs. Neuropathic pain symptoms, classically presenting as 'burning', 'shooting' or 'stabbing', usually reflect peripheral or central neural sensitization and may be associated with allodynia, numbness and diminished thermal sensation. Such symptoms respond poorly to simple analgesics. Monoaminergic, tricyclic and anticonvulsant drugs remain the mainstay of treatment for such vague symptoms. Amitriptyline and selective serotonergic reuptake inhibitors act on descending noradrenergic and serotonergic pathways. Though traditionally used as antidepressants in higher dosage, low-dose treatment is quite beneficial in chronic pain management [20]. Gabapentin and pregabalin were developed as GABA mimetics and are currently licensed for neuropathic pain management. They tend to bind with a higher affinity to the $\alpha_{2} \delta$ subunit of voltage-gated calcium channels at the neuronal level. There is a consistent evidence that they selectively inhibit noxious evoked responses in models of neuropathy and inflammation. Gabapentin has been shown to be effective in the range of $900-1,800 \mathrm{mg} / \mathrm{day}$, though doses up to $3,600 \mathrm{mg}$ daily may be required. Although initial dosing may commence at $300 \mathrm{mg} 3$ times a day in the elderly, it may be preferable to start at just 300 $\mathrm{mg} /$ night. Dose escalation/titration to achieve therapeutic pain relief commonly requires a duration of 3-6 weeks. Pregabalin has a predictable pharmacokinetic profile with a linear dose plasma relationship. It offers an additional advantage of twice-daily dosing and is effective at a starting dose of $75 \mathrm{mg}$ twice daily. However, it is important to understand that there is unpredictable individual

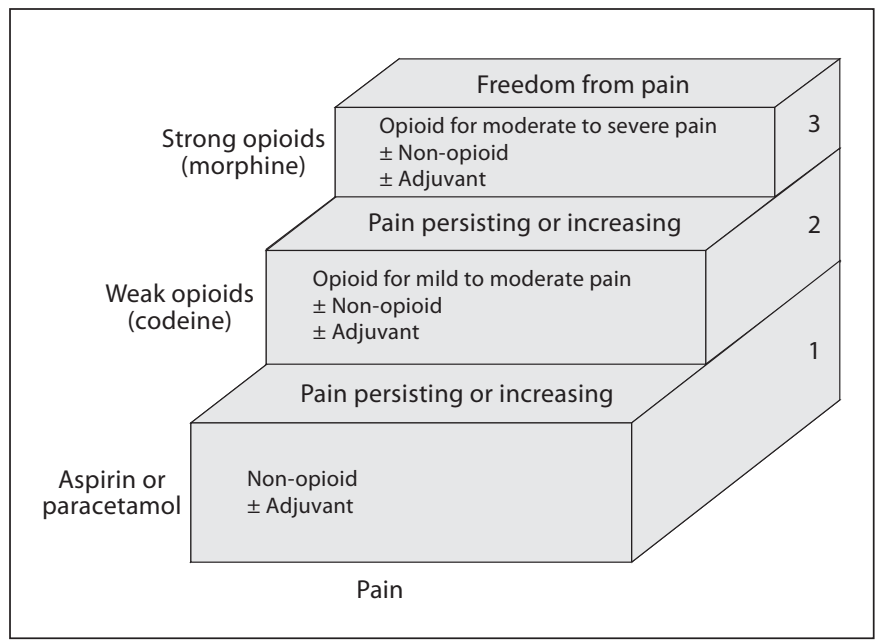

Fig. 1. Three-step analgesic ladder.

variation to the beneficial response to each drug. These drugs have common side effects relating to dizziness, somnolence and dry mouth.

Opioids. The effectiveness of opioids in neuropathic pain is increasingly being recognized. Weak opioids are commonly used with paracetamol and form the second step of the WHO pain ladder. In the past, this has largely been with codeine or dextropropoxyphene, and more recently also with tramadol. The multimodal mechanisms of tramadol make it the first-choice opioid for moderate neuropathic pain. Although rarely indicated in benign testicular pain, unresponsive pain symptoms might ultimately necessitate stronger opioids including morphine and buprenorphine. Various routes and formulations are now available, however these medications are associated with dependence and tolerance and titration should be left to specialized pain units.

\section{Minimally Invasive Interventions}

The genital branch of the genitofemoral and ilioinguinal nerves provide sensory innervation for the anterior scrotal wall. A diagnostic nerve block with a local anesthetic and steroid is quite safe and may provide benefit. If the diagnostic nerve block is successful, it can be either safely repeated or pulsed radiofrequency treatment may be offered [21]. Symptom relief has been noted following blockade of the pelvic plexus via transrectal injection [22].

Pulsed radiofrequency is a new technique wherein a peripherally placed tip of an electrode near the nerves delivers a large current density of $2 \times 10^{4} \mathrm{~A} / \mathrm{m}^{2}$. This cur- 


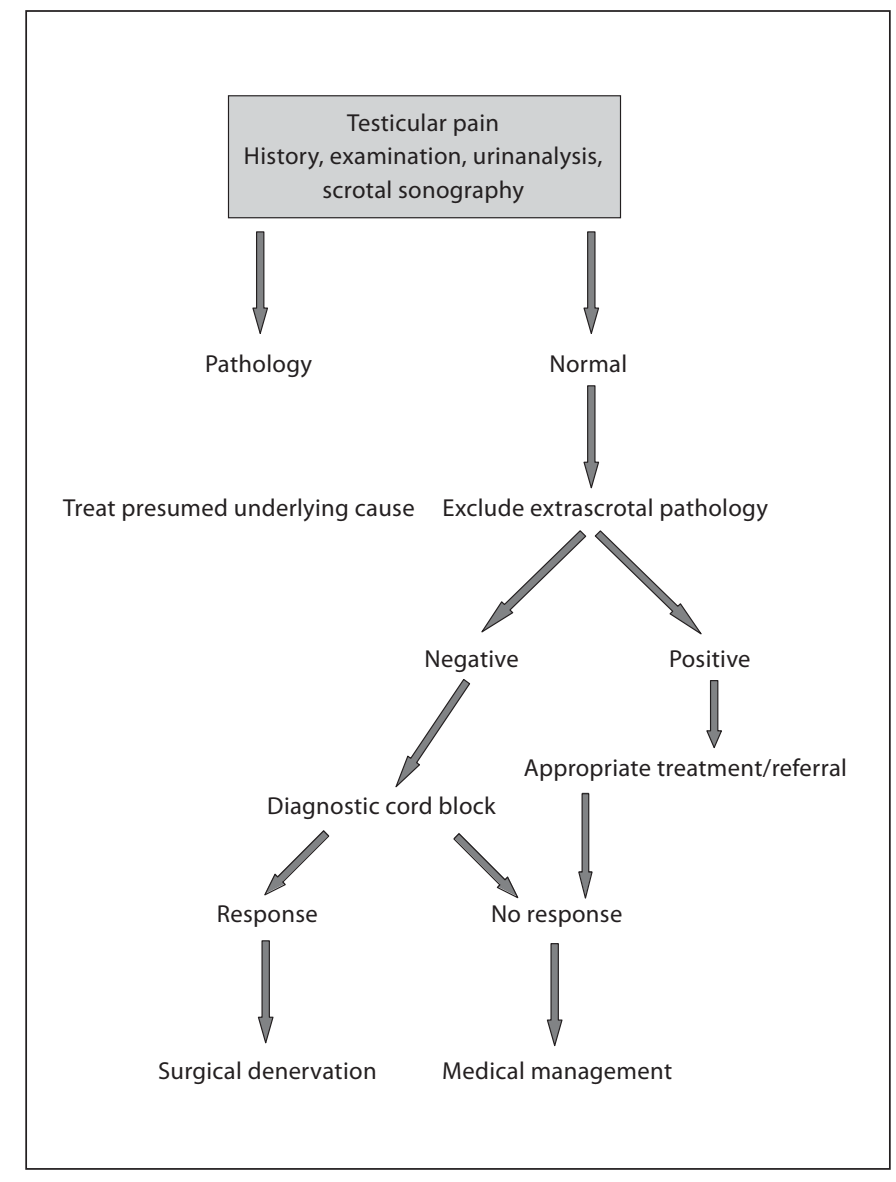

Fig. 2. Algorithm for the management of chronic testicular pain (excluding orchidectomy).

rent can be safely applied to the nerve without either heating it or creating a histologic lesion. A 20-ms pulse of $50,000 \mathrm{~Hz}$ at a frequency of $2 / \mathrm{s}$ is applied for $2 \mathrm{~min}$ and the temperature remains at $<42^{\circ} \mathrm{C}$.

Microsurgical denervation of the cord was first described in 1978 by Devine and Schellhammer [23]. Its aims are to divide all nerve fibers travelling with the spermatic cord. Levine et al. [24] reported their experience with 7 cases and 6 patients had complete resolution of symptoms. They noted a positive correlation between a response to initial cord block and eventual surgical outcome. Cadeddu et al. [25] reported similar results with a transperitoneal laparoscopic approach with 7 of their 9 cases reporting significant improvement in pain. Therefore, if all measures are exhausted and the patient has a response, albeit temporary, to cord blockade, microsurgical denervation may be recommended.

\section{Orchidectomy}

Orchidectomy may be the final operative intervention in these patients. However, $80 \%$ of patients may continue to have orchialgia despite orchidectomy [26]. Orchidectomy should not be rushed into until all other testis-preserving strategies are considered. The patient should also be counseled that despite the testicle being removed he may still have pain. Again it is important to document all the clinical advice given to the patient. An algorithm of management is presented in figure 2 .

\section{Conclusion}

In summary, the approach to the management of chronic orchialgia includes not only the goal of resolution of symptoms but also the mental state of the patient. Relief of symptoms is not always possible and gaining an insight into the patient's concerns and empathizing with their condition is paramount to helping them cope with their symptoms. Extrascrotal etiologies should not be forgotten and therefore a careful assessment is required, sometimes with the help of other specialists. Surgery should not be undertaken lightly for there is no guarantee that there will always be resolution of symptomatology and the patient should be counseled accordingly. Surgery should be reserved for those cases where an underlying abnormality is noted. With the emerging use of microsurgical denervation of the cord, orchidectomy should really be considered the absolute last option.

References 
6 Holland JM, Feldman JL, Gilbert HC: Phantom orchalgia. J Urol 1994;152:2291-2293.

$\checkmark 7$ Robert R, Prat-Pradal D, Labatt JJ, et al: Anatomic basis of chronic perineal pain: role of the pudendal nerve. Surg Radiol Anat 1998; 20:93-98.

$\checkmark 8$ McMahon AJ, Buckley J, Taylor A, et al: Chronic testicular pain following vasectomy. Br J Urol 1992;69:188-191.

9 Guz BV, Ziegelbaum M, Pontes JE: Arteriovenous malformation of spermatic cord. Urology 1989;33:427-428.

10 Naber KG: Management of bacterial prostatitis: what's new? BJU Int 2008;101(suppl 3): 7-10.

-11 Lau MWM, Taylor PM, Payne SR: The indications for scrotal ultrasound. Br J Radiol 1999;72:833-837.

12 Probert JL, Glew D, Gillatt DA: Magnetic resonance imaging in urology. BJU Int 1999; 83:201-214.

13 Watanabe Y, Dohke M, Ohkubo K, et al: Scrotal disorders: evaluation of testicular enhancement patterns at dynamic contrast-enhanced subtraction MR imaging. Radiology 2000;217:219-227.
14 Cramer BM, Schlegel EA, Thueroff JW: MR imaging in the differential diagnosis of scrotal and testicular disease. Radiographics 1991;11:9-21.

15 Gray CL, Powell CR, Amling CL: Outcomes for surgical management of orchalgia in patients with identifiable intrascrotal lesions. Eur Urol 2001;39:455-459.

-16 Rosen MJ, Novitsky YW, Cobb WS, et al: Combined open and laparoscopic approach to chronic pain following open inguinal hernia repair. Hernia 2006;10:20-24.

17 Selikowitz SM, Scned AR: A late post-vasectomy syndrome. J Urol 1985;134:494-497.

$\checkmark 18$ Chen TF, Ball RY: Epididymectomy for postvasectomy pain: histological review. Br J Urol 1991;68:407-413.

19 Reid C, Davies A: The World Health Organization three-step analgesic ladder comes of age. Palliat Med 2004;18:175-176.
20 Sasaki K, Smith CP, Chuang YC, et al: Oral gabapentin (neurontin) treatment of refractory genitourinary tract pain. Tech Urol 2001;7:47-49.

21 Cohen SP, Foster A: Pulsed radiofrequency as a treatment for groin pain and orchalgia. Urology 2003;61:645.

22 Zorn BH, Watson LR, Steers WD: Nerves from pelvic plexus contribute to chronic orchidalgia. Lancet 1994;343:1161.

23 Devine CJ Jr, Schellhammer PF: The use of microsurgical denervation of the spermatic cord for orchialgia. Trans Am Assoc Genitourin Surg 1978;70:149-151.

24 Levine LA, Matkov TG, Lubenow TR: Microsurgical denervation of the spermatic cord: a surgical alternative in the treatment of chronic orchialgia. J Urol 1996;155:10051007.

25 Cadeddu JA, Bishoff JT, Chan DY, et al: Laparoscopic testicular denervation for chronic orchalgia. J Urol 1999;162:733-735.

-26 Constabile RA, Hahn M, McLeod DG: Chronic orchialgia in the pain prone patient: the clinical perspective. J Urol 1991;146: 1571-1574. 